\title{
Tsafon
}

Revue d'études juives du Nord

$75 \mid 2018$

Exil des langues - Langues d'exil

\section{D'une langue à l'autre : exil, identité, écriture chez Olga Grjasnova, Julya Rabinowich et Vladimir Vertlib}

\section{Carola Hähnel-Mesnard}

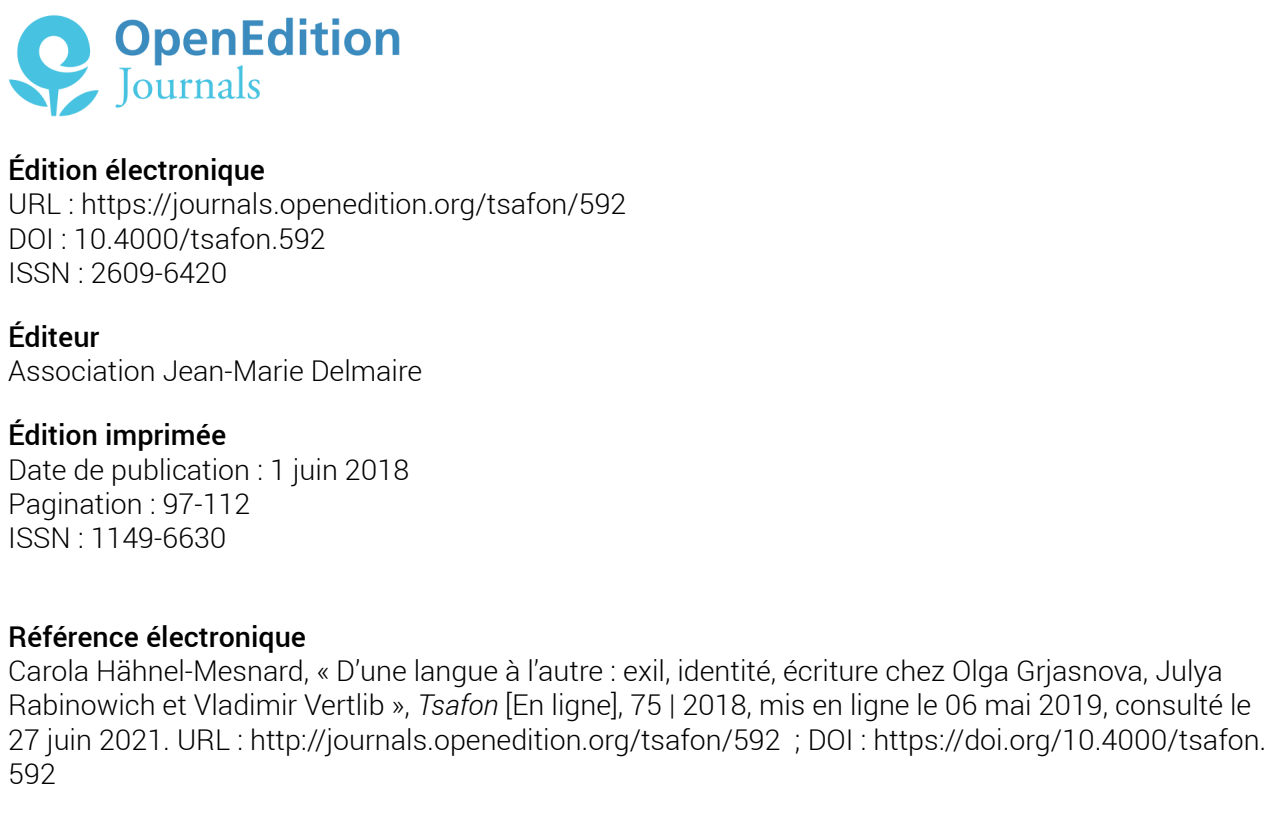

Tsafon. Revues d'études juives du Nord 


\title{
D'une langue à l'autre : exil, identité, écriture chez Olga Grjasnova, Julya Rabinowich et Vladimir Vertlib
}

\author{
Carola Hähnel-Mesnard*
}

Je ne sais pas exactement ce qu'a été ma langue maternelle. Pendant la guerre, il ne fallait pas parler yiddish, langue de mort, mais le français sans accent, puis après la guerre, il fallait passer inaperçu. Ne pas s'appeler Rivka, trop juif, ni AJZERSZTEJN, trop étranger $[\ldots]^{1}$

C'est avec ces réflexions que s'ouvre le dernier livre de Régine Robin, Un roman d'Allemagne, où l'historienne s'interroge sur son rapport à ce pays qui restera pour elle, pour toujours, " une fêlure, une blessure, une béance [...] $»^{2}$. Car si le yiddish, langue maternelle malgré tout, est à tout jamais imprégné de la mort, c'est à cause de cette Allemagne responsable du génocide. Quant au rapport à la langue allemande dont le yiddish est proche, il est complexe: «langue d'Auschwitz » qu'il était interdit de parler à la maison, mais également langue de la culture allemande, langue de Heine dont le père savait réciter les poèmes, langue qui permettait, dans ce que Régine Robin appelle la «distribution fantasmatique des langues ${ }^{3}$, d'établir une filiation imaginaire avec des Juifs allemands pour mieux se distancier de son milieu d'origine de Juifs polonais, considérés comme misérables.

Les exemples de ce rapport complexe et difficile à la langue allemande sont légion dans la génération de ceux qui ont vécu de près la

\footnotetext{
*Université de Lille, SHS.

${ }^{1}$ Régine Robin, Un roman d'Allemagne, Paris, Stock, 2016, p. 11.

${ }^{2}$ Ibid., p. 30.

${ }^{3}$ Ibid., p. 12.
} 
persécution. Mais qu'en est-il des auteurs d'une autre génération qui n'ont pas vécu l'histoire de la même manière ? Le corpus choisi ici concerne des auteurs contemporains juifs d'origine russe qui se sont exilés, avec leurs parents, en Autriche ou en Allemagne dans les années 1970, puis 1990, et qui écrivent désormais en allemand. Alors que leur langue maternelle est le russe, leur rapport à la langue allemande n'est pas dénué de conflits : langue étrangère, langue de l'émigration dont la parfaite maîtrise devient le facteur primordial de leur intégration, langue des bourreaux par rapport à laquelle la génération des parents et des grands-parents - à l'instar de l'exemple évoqué au début - prend ses distances, langue de l'écriture enfin. Et si, pour Régine Robin, écrivain d'une autre génération, le yiddish, «langue dévaluée, dialectique, périphérique $»{ }^{4}$, est le point de départ de son rapport complexe aux langues et à l'allemand en particulier, il devient, pour certains de ces auteurs juifs d'origine russe, comme un point de fuite de leur histoire familiale et un élément qui détermine leur quête d'identité.

Les auteurs dont il sera question ici $^{5}$ font partie de deux générations différentes dont les expériences, malgré beaucoup de similitudes, ne sont pas tout à fait les mêmes. Vladimir Vertlib, né à Leningrad en 1966, et Julya Rabinowich, née également à Leningrad en 1970, quittent l'Union soviétique en pleine Guerre froide et s'installent avec leurs parents en Autriche, à Vienne: Rabinowich en 1977, Vertlib en 1981, après un périple d'une dizaine d'années à travers plusieurs pays. Olga Grjasnowa, née en 1984 à Bakou, vit en Allemagne depuis 1996, sa famille faisait partie de ces réfugiés juifs d'Union soviétique arrivés au début des années 1990. Souvent victimes de discriminations en URSS, ils furent d'abord accueillis par le dernier gouvernement de RDA, avant que cette mesure d'immigration ne fût entérinée par le gouvernement de la République fédérale. On parlait alors de Kontingentflüchtlinge, des réfugiés admis au titre de quotas.

Les trois romans dont il sera question ici sont tous inspirés de la biographie et des expériences d'exil de leurs auteurs, on les caractérisera d'autofictions, basées effectivement sur le vécu autobiographique, mais

\footnotetext{
${ }^{4} \mathrm{Ibid}$.

5 D'autres auteurs ont des vécus d'immigration similaires et profitent d'une reconnaissance littéraire en Allemagne, comme Wladimir Kaminer, Lena Gorelik et plus récemment Sasha Marianna Salzmann. Nous nous limitons toutefois aux trois auteurs choisis qui expriment de façon très pertinente le rapport entre exil, langue et identité.
} 
recourant largement à la fiction pour se confronter notamment aux

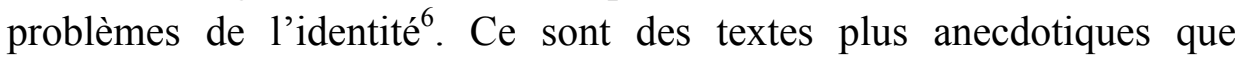
réflexifs, mais avec des intrigues suffisamment explicites pour en tirer quelques conclusions sur le rapport entre langue et exil. Dans un premier temps, il s'agira de retracer l'expérience de l'exil et le rapport aux différentes langues rencontrées, notamment à l'allemand, pour, dans un deuxième temps, interroger la façon dont les auteurs - ou leurs protagonistes - essayent de renouer avec leurs racines en s'intéressant à leur histoire familiale à partir des expériences faites pendant l'émigration.

\section{Exil, langue(s) de l'exil et écriture}

Dans les textes des trois auteurs, les expériences avec la langue du pays d'accueil se ressemblent: les enfants ont d'abord des difficultés scolaires avant de réussir brillamment, ils apprennent plus vite que leurs parents et les assistent pour régler les nombreuses formalités administratives, ayant ainsi un rôle de traducteurs et de médiateurs entre les cultures. Ils font l'expérience de l'exclusion, sont traités d'étrangers, insultés et observent leurs parents dans des situations humiliantes.

Pour Vladimir Vertlib, l'expérience de l'émigration s'étend sur une dizaine d'années et sur différents pays : Israël, l'Autriche, l'Italie, les Pays-Bas et les États-Unis, avant que la famille ne s'installe définitivement en Autriche. Les langues rencontrées sont donc multiples, et rien ne laisse présager qu'un jour, il écrira en allemand. Son premier roman, Zwischenstationen [Étapes intermédiaires] (1999), relate ce périple et la relation complexe à différentes langues. Avant d'entrer en contact avec l'allemand qui deviendra, pour l'auteur, également la langue de l'écriture, le protagoniste du roman se confronte à l'hébreu, qui reste difficile pour lui. Arrivé à Vienne quelques années plus tard, l'hébreu sera, à côté du russe, la langue de communication dans leur nouvel immeuble qui « était une partie d'Israël et de la Russie se trouvant dans

\footnotetext{
${ }^{6}$ Chiara Conterno recourt au concept d'autofiction dans son analyse des œuvres de Vertlib et de Rabinowich, mais il peut également s'appliquer à Grjasnowa ou à d'autres auteurs de ce «courant». Nous sommes plus réservée quant à son utilisation quelque peu inflationniste du concept de «trauma», voire de «traumas multiples» dans ce contexte. Cf. Chiara Conterno, "Trauma multipli. Zwischenstationen di Vladimir Vertlib e Spaltkopf di Julya Rabinowich », LEA - Lingue e letterature d'Oriente e d'Occidente, $\mathrm{n}^{\circ} 2 / 2013$, p. 269-283, p. 278 pour l'autofiction.
} 
un monde étranger appelé Vienne ${ }^{7}$, et il apparaît comme la langue qui relie le narrateur à ses racines, à la religion de ses ancêtres. En effet, un vieux Juif pratiquant, ami de la famille, se plaint de l'ignorance du jeune homme en matière de religion juive, et lui propose de lui apprendre l'hébreu, « la plus ancienne langue vivante au monde $»^{8}$.

L'allemand arrive ensuite, sous forme d'injures prononcées à l'encontre des immigrés. C'est à l'école que le narrateur apprend finalement l'allemand et l'institutrice lui fait le redoutable compliment d'être meilleur que «ces Turcs » ${ }^{9}$. Arrivé au stade où cette dernière lui atteste que le plus difficile est derrière lui, la famille est sur le point de quitter Vienne et de remettre en cause le fragile équilibre que le narrateur a instauré avec l'allemand. Le séjour aux États-Unis s'accompagne de l'apprentissage rapide d'une nouvelle langue dans laquelle il excelle rapidement, traduisant les lettres que son père adresse aux autorités pour demander le droit de séjour en les modifiant et en les fictionnalisant à l'envi, moments pendant lesquels il ressent un grand sentiment de bonheur $(Z S, 227)$. Pour certains interprètes, cela anticipe le passage à l'écriture du personnage (et de l'auteur) qui transforme plus tard dans ses livres la réalité vécue en fiction ${ }^{10}$; pour d'autres, l'élément déclenchant de sa carrière d'écrivain se situe avant, lorsque, encore en Autriche, il trouve par hasard un exemplaire des Souffrances du jeune Werther de Goethe grâce auquel il pourra ensuite briller en cours d'allemand et rejoindre le monde des intellectuels ${ }^{11}$.

Dans l'ensemble, le parcours du narrateur décrit dans cette fiction ressemble à une intégration et à une assimilation parfaites. En quittant Vienne à la fin du récit pour rejoindre son amie à Salzbourg, le narrateur va jusqu'à s'acheter un chapeau tyrolien, comme s'il voulait se convaincre qu'il fait à présent bien partie de cette société autrichienne. Selon Alfred Strasser, le roman de Vertlib va plus loin que beaucoup de romans sur la migration, dans la mesure où il compare différents lieux de

\footnotetext{
${ }^{7}$ Vladimir Vertlib, Zwischenstationen [1999], München, dtv, 2005, p. 31: « Das Haus war ein Teil Israels und Rußlands, der sich in einer fremden Welt namens Wien befand». Abrégé par la suite par $Z S$ avec numéros de page dans le texte. Nous traduisons.

8 "Hebräisch ist die älteste lebende Sprache der Welt, dreitausend Jahre alt ». (ZS, 272).

${ }^{9}$ « Die Lehrerin lobt mich oft, daß ich besser bin als diese Türken ». (ZS, 59).

${ }^{10}$ Cf. Adrian Wanner, Out of Russia: Fictions of a New Translingual Diaspora, Evanston, Illinois, Northwestern University Press, 2011, p. 83 sq.

${ }^{11}$ Cf. Sander L. Gilman, "Becoming a Jew by Becoming a German: The Newest Jewish Writing from the 'East' », Shofar: An Interdisciplinary Journal of Jewish Studies, vol. 25, n 1/2006, p. 16-32, ici p. 29.
} 
migration, donc différents « ailleurs », et dans la mesure où le narrateur, et avec lui l'auteur, se serait rendu compte que ce qui lui était propre lui est devenu étranger, et qu'il s'est approprié l'altérité en choisissant l'allemand comme langue d'écriture ${ }^{12}$.

En réalité, le rapport de l'auteur à la langue allemande est bien plus complexe, comme il l'explique dans ses conférences poétiques publiées en 2007 dans le volume Spiegel im fremden Wort [Reflets dans la parole étrangère]. Tout d'abord, le rapport à l'allemand a été fortement marqué par l'attitude du père qui « n'aimait pas l'allemand », « la langue d'Adolf Hitler $»^{13}$, et qui a toujours refusé de l'apprendre, sans réfléchir, comme le commente l'auteur, à la proximité entre l'allemand et le yiddish qui était, pendant des générations, la langue de ses ancêtres ${ }^{14}$. Pour Vertlib, le chemin à parcourir pour maîtriser cette nouvelle langue - condition préalable à l'intégration - fut donc d'autant plus pénible que son père s'y opposa. «Il n’y avait pas de relation amoureuse spontanée et impulsive entre moi et la nouvelle langue », mais plutôt un « mariage de raison ${ }^{15}$, une relation pragmatique, explique l'auteur. Écrire en allemand n'allait pas de soi non plus, les premières lettres d'amour s'écrivent en anglais, et les premières tentatives d'écriture se faisaient en russe où les mots avaient «un sens profond» alors qu'en allemand, ils avaient une "signification »". Aujourd'hui, l'auteur décrit son écriture comme un mélange entre « une surface allemande en dessous de laquelle résonnent [...] la syntaxe, la mélodie et les expressions idiomatiques du russe $\gg^{17}$.

\footnotetext{
${ }^{12}$ Alfred Strasser, « Einmal Leningrad - Wien - New York und zurück. Stationen einer Odyssee in Vladimir Vertlibs Roman Zwischenstationen ", Germanica [Online], 38/2006, consulté le $1^{\text {er }}$ octobre 2016. URL: http://germanica.revues.org/378, p. 8 : « Schließlich stellt er [Vertlib] fest, daß ihm das Eigene fremd geworden ist und er sich das Fremde angeeignet hat, was vor allem auch in der Wahl der Sprache, in der er den Roman verfaßt hat, zum Ausdruck kommt ».

${ }^{13}$ Vladimir Vertlib, Spiegel im fremden Wort. Die Erfindung des Lebens als Literatur, Dresdner Chamisso-Poetikvorlesungen 2006, Dresden, Thelem, 2007, p. 52.

${ }^{14}$ Ibid.. On observe des réflexions similaires à celles de Régine Robin évoquées dans l'introduction, mais sans l'insistance sur le caractère "périphérique » du yiddish: «Interessanterweise spielte es für ihn keine Rolle, dass Dutzende Generationen seiner Vorfahren Jiddisch, eine dem Deutschen sehr ähnliche Sprache, gesprochen hatten. Keine andere Sprache ist dem Deutschen so nahe wie Jiddisch ».

15 Ibid., p. 53 : «Und dann [...] kam es zu keiner spontanen, impulsiven Liebesbeziehung zwischen mir und der neuen Sprache. Unsere Beziehung ist vielmehr wie eine Ehe, die aus pragmatischen Gründen geschlossen wurde [...]».

${ }^{16}$ Ibid., p. 58 : «Im Deutschen hatten die Worte eine Bedeutung, im Russischen, meiner Muttersprache, einen tieferen Sinn ».

${ }^{17}$ Ibid., p. 59 : « [...] eine deutsche Oberfläche, unter der oft [...] Satzbau, Melodie und Idiomatik des Russischen mitschwingen ».
} 
Ce que Vertlib dit ensuite à propos de la Sprachheimat, la langue dans laquelle il se sent chez lui, est également révélateur. Il considère le plurilinguisme non pas uniquement comme un atout, mais également comme une perte, désignant par là les confins des langues où l'on reste monolingue, soit par incapacité, soit par proximité émotionnelle à une seule langue qui interdit l'utilisation de l'autre ${ }^{18}$. Ces remarques qui éclairent le rapport de l'auteur à la langue étrangère dans son écriture entrent également en résonance avec les réflexions de Yasemin Yildiz sur la «condition post-monolingue ». Si Yildiz plaide pour le dépassement du paradigme du monolinguisme, largement dominant dans nos sociétés qui voient pourtant émerger de plus en plus les pratiques plurilingues, elle interroge tout d'abord, à l'exemple de plusieurs auteurs, les tensions historiques, mais aussi personnelles, entre le mono- et le plurilinguisme $^{19}$.

Julya Rabinowich, notre deuxième auteure, a fait partie de la même vague d'immigration que Vladimir Vertlib mais a vécu un périple moins chaotique, et son rapport à la langue allemande semble un peu moins compliqué. À l'instar du protagoniste du roman de Vertlib, la narratrice de Spaltkopf [Tête fendue] (2008), métaphore pour cette existence entre deux langues et deux cultures, a dû se battre pendant sa scolarité pour qu'on reconnaisse ses particularités et qu'on ne la considère plus comme exotique. Elle emploie tous les moyens pour parler très rapidement l'allemand mieux que les autres enfants d'immigrés, turcs ou yougoslaves, jusqu'à ce qu'un jour sa pratique corresponde à celle de la meilleure élève de la classe. On retrouve les thèmes déjà traités par Vertlib, avec une focalisation plus importante sur l'adolescence, le sortir de l'enfance que la narratrice vit difficilement.

Or, ce n'est pas tant la langue allemande qui semble réellement poser problème à la jeune Mischka, protagoniste et narratrice de ce premier roman, mais une absence de parole qui s'empare de plus en plus de la famille. L'exil, c'est aussi la désintégration de la famille sous la pression des nouvelles conditions de vie, ce sont les désillusions dans ce nouveau monde suivies d'un mutisme de plus en plus pesant. Dès lors, la naissance d'un deuxième enfant - sensé sauver le couple des parents qui reste muet et bloqué dans son développement, apparaît comme la

\footnotetext{
${ }^{18}$ Ibid., p. 60 sq.

${ }^{19}$ Yasemin Yildiz, Beyond the Mother Tongue. The Postmonolingual Condition, New York, Fordham University Press, 2012.
} 
parabole de cet exil désenchanté : «Le mutisme s'étend. [...] Nous nous défendons tous contre la langue, nous sommes tous à bout de souffle. Quand il n'y a plus d'actions, les mots font défaut. Nous devenons muets $»^{20}$. C'est à ce moment précis que Mischka sort une vieille machine à écrire pour composer son premier texte et sortir de ce silence familial. Silence qui renvoie également à une autre absence de parole derrière laquelle se cache un secret familial, révélé peu à peu, au fil du récit. Dans le roman, c'est l'écriture qui semble être à même de dévoiler les secrets de famille. Quant au russe, langue maternelle du personnage, il réapparaît dans ses rêves après la naissance de sa propre fille, mais cette langue s'avère être "encombrante», elle se "coince dans sa bouche comme du bois flottant», provoque des «sentiments récalcitrants » qui érigent des «barricades» entre elle-même et sa nouvelle vie viennoise, ironiquement comparée à un petit jardin familial synonyme d'un certain confort modeste ${ }^{21}$. Cette réapparition soudaine et inattendue du russe, comme quelque chose de refoulé, s'accompagne d'un manque de parole pour désigner ces sentiments contradictoires. De même, lorsque la narratrice se rend en Russie pour la première fois depuis son émigration, elle reconnaît des odeurs et ne peut s'en souvenir que «sans paroles ${ }^{22}$; le passé réapparaît sous forme d'objets, de sens, mais la capacité à le nommer est perdue. L'allemand semble avoir effacé la langue maternelle, mais en réalité, c'est le russe qui représente depuis longtemps le silence et un secret familial que le personnage va mettre au jour grâce à son écriture en allemand, nous y reviendrons.

Quant à l'auteure, elle affirme que c'est précisément la langue allemande qui lui permet de prendre ses distances avec son vécu, accordant à la langue étrangère une fonction presque cathartique. Interrogée sur la teneur autobiographique de son premier livre, elle affirme que c'est grâce à l'allemand qu'elle arrive à s'émanciper des personnages qui peuplent sa vie réelle ${ }^{23}$. C'est dans la langue, et plus

\footnotetext{
${ }^{20}$ Julya Rabinowich, Spaltkopf (par la suite abrégé par $S$ suivi des numéros de page), Wien, Deuticke, 2011, p. 116 : «Sprachlosigkeit breitet sich aus. [...] Wir alle wehren uns gegen die Sprache, uns allen geht die Luft aus. Wenn keine Taten mehr bleiben, fehlen die Worte. Wir verstummen ».

${ }^{21}$ «Ich träume auf Russisch neuerdings. Ich spüre, wie sich die sperrige Sprache in meinem Mund verkeilt wie Treibholz, wie widerborstige Gefühle Barrikaden errichten zwischen mir und meinem Wiener Schrebergärtchen ». (S, 160).

22 « Die Küche duftet nach Dingen, an die ich mich nur wortlos erinnere. » (S 190).

23 ",Abgründe haben mich immer angezogen“. Julya Rabinowich im Gespräch mit Ernst Grabovszki », Wiener Zeitung, 21 mars 2009. Consulté le 5/11/2016 sur:
} 
particulièrement dans la langue allemande, qu'elle se sent à la fois « arrivée » et « chez elle $»^{24}$. En cela, les propos de l'auteure vont à l'encontre d'un fait communément accepté pour la «littérature interculturelle » selon lequel le contenu souvent autobiographique de ces textes permettrait aux auteurs une certaine assise, un point d'appui face au processus d'écriture qui se fait dans une autre langue ${ }^{25}$. Ici, au contraire, c'est l'autre langue qui libère la parole sur le vécu.

Olga Grjasnowa, née à Bakou en 1984 et arrivée en Allemagne en 1996, publie un premier roman en 2012 sous le titre Der Russe ist einer, der Birken liebt [Le Russe aime les bouleaux $]^{26}$. La narratrice et protagoniste Mascha maîtrise de nombreuses langues et s'apprête à faire une carrière d'interprète à l'ONU. D'origine russe et juive, elle arrive en Allemagne à l'âge de onze ans et fait, elle aussi, tout d'abord, l'expérience de l'exclusion et d'une scolarité difficile. En accompagnant ses parents régler les diverses formalités administratives, elle comprend très vite que «les langues signifiaient le pouvoir », que «ceux qui ne parlaient pas allemand n'avaient pas de voix» et que les diverses « demandes étaient traitées en fonction de la gravité de l'accent ${ }^{27}$. Forte de ces expériences discriminantes, Mascha étudiera plus tard les langues de façon presque boulimique, elle en maîtrise au moins cinq couramment, d'autres de façon plus rudimentaire. Mais lorsqu'elle veut aller plus loin dans ses études d'interprète, elle se voit reprocher par un

(https://web.archive.org/web/20100117182244/http://www.wienerzeitung.at/Desktop Default.aspx?TabID=4664\&Alias=Wzo\&cob=404059).

24 «In der Sprache allerdings fühle ich mich sowohl angekommen als auch daheim». " „Dann hätten wir bald viele Würstelstand-Literaten“. Gespräch mit Julia Schilly », Der Standard, 19/11/2008. derstandard.at/ 1226396889022/Interview-Dann-haettenwir-bald-viele-Wuerstelstand-Literaten. Dernier accès : 5/11/2016.

${ }^{25} \mathrm{C}$ 'est toutefois la thèse défendue par Natalia Blum-Barth dans sa recension du roman qui ne semble pas tenir compte du potentiel familial conflictuel que le pays d'accueil et la langue allemande tiennent à distance. Cf. N. Blum-Barth, " „Spaltkopf“ als interkultureller Roman. Zur Neuauflage des Romans „Spaltkopf“ von Julya Rabinowich », http://literaturkritik.de/public/rezension.php?rez_id=15957. Dernier accès : 16/02/2018.

${ }^{26}$ Olga Grjasnowa, Der Russe ist einer, der Birken liebt [2012], München, dtv, 2013. Abrégé par la suite par $R B$ suivi du numéro de page. C'est le seul roman de notre corpus traduit en français: Le Russe aime les bouleaux, traduit de l'allemand par Pierre Deshusses, Paris, Les escales éditions, 2014.

27 « Ich begleitete meine Eltern zum Ausländeramt und lernte dort, dass Sprachen Macht bedeuteten ». $(R B, 37)$; «Wer kein Deutsch sprach, hatte keine Stimme [...]. Anträge wurden entsprechend der Schwere der Akzente bewilligt». $(R B, 38)$. Nous traduisons. 
professeur «pratiquant son multiculturalisme dans des salles de conférences » ( $R B$ 33), qui est au courant de ses racines russes et azerbaïdjanaises, que son plurilinguisme a été acquis sur le tard, qu'elle n'était pas une vraie locutrice native, rappelant un autre versant de ce que Yasemin Yildiz avait appelé le paradigme du monolinguisme, notamment l'idée de l'essentialisation de la «langue maternelle $»^{28}$. Or, elle avait appris l'azéri non pas par ses parents, mais au contact de ses voisins et elle le parlait de façon fluide et sans accent jusqu'à ce qu'elle émigre en Allemagne. D'ailleurs, son meilleur ami, Cem, un Turc avec qui elle parle généralement allemand comme «deux étrangers modèles parfaitement intégrés » $(R B 57)$, peut lui raconter les anecdotes de son enfance en turc, et elle les siennes en azéri, dans la mesure où les deux langues sont très proches.

Dans l'univers polyglotte de la narratrice, la langue de l'exil ne semble désormais plus être problématique. De même, pour l'auteure, l'allemand est la seule langue qu'elle ressent et qu'elle maîtrise avec assurance, bien qu'elle parle encore couramment le russe ${ }^{29}$. Or, le choix de s'installer en Allemagne, et donc de se confronter à la langue allemande, n'allait absolument pas de soi pour cette famille de Juifs russes dont la grand-mère était une survivante du génocide. On retrouve ici la constellation de départ de Vladimir Vertlib. Au début, les parents trouvaient absurde l'idée de partir en Allemagne, mais c'était, à côté d'Israël, le seul pays à accepter sans problème les Juifs soviétiques. L'émigration de la famille n'était d'ailleurs pas due à ses origines juives, mais à la situation conflictuelle dans la région du Haut-Karabagh où Arméniens et Azéri s'affrontaient et où des pogroms anti-arméniens faisaient de nombreuses victimes au début des années 1990. Dans ce contexte, le grand-père paternel de la narratrice est pris pour un Arménien et attaqué, il en meurt trois jours après d'une crise cardiaque. Sa petite-fille, Mascha, devient témoin des violences, avec un cadavre défenestré qui lui tombe littéralement devant les pieds, et la grand-mère maternelle, Juive survivante, ne peut que constater que « tout se répète » ( $R B$ 283). Dire, comme le fait la narratrice au début de son récit, que leur émigration n'a rien à voir avec les racines juives de la famille, ignore donc a priori les traumatismes profonds qui la marquent. Alors qu'elle

\footnotetext{
${ }^{28}$ Y. Yildiz, Beyond the Mother Tongue..., op. cit., p. 6-14.

29 Voir 1'entretien filmé «Olga Grjasnowa: Russisch ist meine Muttersprache trotzdem schreibe ich lieber auf Deutsch», http://interview-lounge.tv/olga-grjasnowaim-gesprach/. Dernier accès : 16/02/2018.
} 
semble elle-même largement ignorer ses racines, Mascha les découvrira suite à un drame personnel, la mort de son compagnon, qui déclenche une crise et une recherche de son identité.

\section{Langue, identité et histoire familiale}

Pendant longtemps, la narratrice du roman de Grjasnowa n'a qu'un rapport très éloigné avec le judaïsme. Mais lorsqu'elle craint de perdre son ami suite à un accident, elle se souvient des deux prières qu'elle connaissait, « Notre père » et "Chema Israël». Or, n'ayant aucune connaissance authentique des pratiques religieuses, elle va s'inspirer de la télévision pour mettre en pratique la prière juive, elle «se balançait de la même manière que les Juifs orthodoxes vus sur Arte ${ }^{30}$. En revanche, après le décès de son compagnon, Mascha, à la recherche d'un ancrage, s'intéressera de plus en plus à la religion juive et au Talmud. Pour faire son deuil, elle choisit de s'installer en Israël, entamant ainsi une quête des racines qui va la confronter à ses identités multiples. Dès son arrivée à Ben Gourion, Mascha entend une multiplicité de langues dont la plupart lui sont familières, leurs «mélodies se mélangent en un fond sonore : le russe, l'hébreu, l'anglais, l'italien et l'arabe $»^{31}-1$ 'allemand Klangteppich, littéralement «tapis sonore » suggérant ici une sorte de tapis rouge des langues qui se déroule devant elle pour l'accueillir et la faire se sentir chez elle, dans un univers plurilingue. Comme l'a fait remarquer Adrian Wanner, la protagoniste semble avoir un avantage par rapport à d'autres émigrants, celui de n'avoir aucune difficulté à apprendre de nouvelles langues. En même temps, ces langues ne l'aident pas à se trouver elle-même, elles sont comme une interminable quête pour trouver un «chez soi », une Heimat, que la protagoniste n'a jamais eue $^{32}$. Dans cette perspective, le choix de son métier est également significatif, car en tant qu'interprète, elle devient une médiatrice culturelle tout en renonçant à sa propre voix, elle répète ce que disent les

\footnotetext{
30 «Ich wiegte mich im Gebet, wie ich es bei den orthodoxen Juden auf Arte gesehen hatte $» .(R B, 24)$.

31 «[...] in der Flughafenhalle vermischten sich die Sprachmelodien zu einem Klangteppich: Russisch, Hebräisch, Englisch, Italienisch und Arabisch ». $(R B, 161)$.

32 «Her compulsive learning of new languages looks like a desperate attempt to find the 'home' that she never had in the first place ». Cf. Adrian Wanner, "Writing the Translingual Life: Recent Memoirs and Auto-Fiction by Russian-American and Russian-German Novelists », L2 Journal, vol. 7, 2015, p. 141-151, ici p. 148. (https://escholarship.org/uc/item/85t9d1xh).
} 
autres sans jamais parler d'elle-même ${ }^{33}$. Son voyage en Israël est une tentative de s'ancrer dans son histoire en tant que juive, et peut-être d'approcher l'hébreu sous un angle moins superficiel, loin des automatismes d'une prière apprise quand elle était enfant.

Or, le premier contact avec le pays est loin d'être aussi chaleureux que le fond sonore des langues qui l'accueille : son ordinateur est détruit à cause de caractères arabes collés sur le clavier, puis la cousine lui ayant suggéré de faire son alya lui dit encore à l'aéroport qu'elle n'a pas l'air juif et qu'elle ne lui ressemble pas, lui fermant d'emblée la porte pour intégrer sa famille ${ }^{34}$. Puis Mascha se voit continuellement reprocher de parler couramment l'arabe, mais non pas l'hébreu. Provenant d'une « famille orientale» $(R B, 10)$, comme elle le souligne, elle a eu plus de proximité avec l'azéri et le turc d'un côté, et l'arabe, de l'autre, son premier ami étant Libanais. Mais l'arabe lui semble surtout primordial dans une perspective professionnelle : « À quoi cela sert d'apprendre une langue aussi petite que l'hébreu, alors que je peux avoir une langue de l'ONU ? $\gg{ }^{35}$. Si la protagoniste répond en mettant en avant la seule utilité, considérant l'hébreu comme langue mineure dont elle semble ignorer toute la dimension culturelle et religieuse - comme d'ailleurs le protagoniste de Zwischenstationen de Vertlib -, c'est aussi une réaction de défense. L'hébreu apparaît comme un «blanc » dans le parcours de la protagoniste, une place laissée vide non seulement dans la cartographie des langues qu'elle maîtrise mais également dans sa quête d'identité. L'accueil qui lui est fait l'éloigne de prime abord de cette langue.

Par un enchaînement de circonstances, Mascha se retrouve en Cisjordanie, dans le camp de réfugiés de Jénine. Se retrouvant seule dans la ville où elle aperçoit des affiches avec des auteurs d'attentats suicides et des croix gammées, son propre traumatisme surgit : les violences antiarméniennes dont elle a été témoin à Bakou, sa mère qui lui enlève la petite chaîne avec l'étoile de David qu'elle porte depuis l'âge de trois ans, les paroles de sa grand-mère comme quoi l'histoire se répète, son juron en yiddish lorsqu'un Azéri la soupçonne de cacher des Arméniens. Pour la protagoniste, Israël ne sera finalement pas le lieu qui effacera ses

\footnotetext{
${ }^{33}$ Ibid.

34 Voir à ce sujet également Paula Wójcik, «Identität in Transgression - Olga Grjasnowas „Der Russe ist einer, der Birken liebt“", Medaon. Magazin für jüdisches Leben in Forschung und Bildung, 9/2015, 16, p. 1-12. En ligne: http://www.medaon.de/pdf/medaon_16_Wójcik.pdf. Dernier accès : 17/02/2018.

35 «Was macht es für einen Sinn, eine so kleine Sprache wie Hebräisch zu lernen? Wenn ich eine UN-Sprache haben kann? ». $(R B, 189)$.
} 
traumatismes, bien que Tel-Aviv lui rappelle sa ville natale qui reste, malgré les souvenirs douloureux, son «chez-soi ». Le conflit israélopalestinien a ravivé les mauvais souvenirs d'enfance, Israël apparaît comme un pays qui réclame d'elle un engagement ferme pour lequel elle n'est pas prête, ayant compris aussi que la seule langue qu'elle ne maîtrise pas est celle qui fonde l'identité du pays.

La quête d'identité de la protagoniste du roman d'Olga Grjasnowa, évoluant sur l'axe Azerbaïdjan - Allemagne - Israël, est donc fortement déterminée par son rapport à différentes langues qui ne sont pas sa langue maternelle, le russe ne jouant d'ailleurs presque plus de rôle tout au long du roman. Dans cette constellation, l'hébreu apparaît comme un point de fuite qui s'éloigne dès que la narratrice s'en approche, la rejetant dans son monde plurilingue, mais sans véritables attaches. Elle finira par accepter que l'Allemagne soit le lieu le mieux adapté pour vivre son identité hybride.

Vladimir Vertlib et Julya Rabinowich retracent également la quête de leurs racines juives, non pas par l'intermédiaire d'Israël et de l'hébreu, mais en faisant des recherches sur l'origine et le vécu de leurs grandsparents. Chez Vladimir Vertlib, la situation de l'exil dans plusieurs pays, puis en Autriche, déclenche une réflexion sur le rapport aux langues dans sa famille. Alors que ses parents ont grandi en Union soviétique avec une seule langue et une seule culture, le russe, ses aïeux ont vécu pendant des siècles au carrefour de plusieurs cultures et parlaient à la fois le yiddish, le russe, le biélorusse, l'ukrainien, le polonais, l'allemand et l'hébreu, appris au heder et à la synagogue ${ }^{36}$. Pour les parents, les langues étrangères et tout ce qui était étranger n'étaient «qu'un souvenir enfoui de leurs propres racines juives, une intuition, une illusion $»^{37}$. L'assimilation de la génération des grands-parents a entraîné la perte de ce plurilinguisme et surtout, comme le souligne Vertlib, celle du yiddish : alors que ses grands-parents parlaient encore le yiddish avant la guerre, l'usage a peu à peu été abandonné, entraînant la perte de cette langue et son oubli ; à la fin de sa vie, sa grand-mère ne comprenait même plus le yiddish, sa langue maternelle ${ }^{38}$.

\footnotetext{
${ }^{36}$ Cf. V. Vertlib, Spiegel im fremden Wort, op. cit., p. 49.

${ }^{37}$ Ibid. : « Der Fremdsprachenunterricht in der Schule war ein Witz, das Fremde eine verschüttete Erinnerung an die eigenen jüdischen Wurzeln, eine ungefähre Ahnung, eine Illusion $\gg$.

${ }^{38}$ Ibid., p. 50.
} 
Afin d'en savoir plus sur cette culture, sur la vie de ses arrièregrands-parents avant 1917, sur la vie au shtetl à cette époque et les racines polyglottes de sa famille, Vertlib a mené des entretiens avec sa grand-mère qu'il enregistre sur des cassettes audio. Si les récits de famille, les anecdotes transmises par les parents, étaient pour lui une sorte de Heimat, un monde à part qui servait de refuge pendant les années de l'exil et permettait de s'échapper du quotidien de l'émigrant, l'absence de sa grand-mère, restée en Union soviétique, représente pour l'enfant une sorte «d'expulsion du jardin Éden de la grande famille » ${ }^{39}$. Les retrouvailles, presque vingt ans plus tard, et les conversations avec cette grand-mère sont pour lui une étape importante dans sa quête de soi. Mais il a fallu quinze ans pour que l'auteur se sente assez mûr avant d'utiliser ces récits enregistrés pour en faire un roman. Das besondere Gedächtnis der Rosa Masur [L'étrange mémoire de Rosa Masur] ${ }^{40}$, sorti en 2001, relate la vie d'une femme juive, russe, à travers le siècle, en accordant une grande importance au siège de Leningrad, lui valant la comparaison avec Joseph Roth et Isaac Singer ${ }^{41}$.

L'exil, le passage par différentes langues et cultures éveillent donc chez Vertlib le désir d'en savoir davantage sur les origines de sa famille, sur cette culture anéantie du shtetl, sur le yiddish, puis le vécu de sa grand-mère juive en Union soviétique à travers les décennies. Ces récits de vie seront transposés en langue allemande, langue de l'écriture, qui devient ici un vecteur de transmission de cette mémoire juive.

Julya Rabinowich, quant à elle, invente dans son roman Spaltkopf une voix narrative particulière qui se fait chroniqueuse de l'histoire familiale et, notamment, de ses secrets et expériences refoulés. Alors que la narratrice évoque le monde de son enfance, l'émigration, puis la vie en Autriche, une deuxième instance narrative commente les paroles de la narratrice, y ajoute des informations, rectifie et ajuste. Ces passages sont écrits en italiques, sur un mode fragmentaire, elliptique et avec un ton souvent poétique, presque mystique ${ }^{42}$. Cette voix est supposée être celle

\footnotetext{
39 Ibid., p. 92 : «Die Emigration war eine Vertreibung aus dem Paradies der Großfamilie ».

${ }^{40}$ Il s'agit du seul livre de Vertlib actuellement traduit en français: V. Vertlib, L'étrange mémoire de Rosa Masur, traduit de l'allemand par Carole Fily, Paris, Métailié, 2016.

${ }^{41}$ Cf. Alexander Kissler, «Miss Jahrhundert. Vladimir Vertlibs Rejsele erzählt », Frankfurter Allgemeine Zeitung, 23/06/2001.

${ }^{42}$ Cf. N. Blum-Barth : « „Spaltkopf“" als interkultureller Roman... », op. cit.
} 
du Spaltkopf, de la «tête fendue » qui donne son titre au roman. Cette « tête fendue » joue également un rôle dans le récit principal, elle y est présentée comme si elle provenait d'un conte russe, c'est une sorte d'esprit malveillant qui dévore les pensées des enfants et les vide de leur âme quand ils refusent de dormir. En réalité, ce conte n'existe pas, la «tête fendue » ne fait pas partie de la mémoire culturelle, mais elle est "privée et faite sur mesure», selon l'expression utilisée par Silke Schwaiger, c'est une invention de l'auteure ${ }^{43}$. Dans l'économie du roman, cette « tête fendue » se dédouble, comme son nom l'indique, elle devient métaphore de l'inconscient et du refoulé de l'histoire familiale, des mauvais souvenirs dont on veut se départir, une métaphore de l'oubli ${ }^{44}$.

Ce refoulé concerne notamment l'identité juive de la grand-mère maternelle que celle-ci renie depuis qu'elle a vécu un traumatisme pendant son enfance. Dans les années 1920, avec ses parents, elle est victime d'un pogrome lors duquel son père est tué, alors qu'elle et sa mère survivent. Adulte, elle décidera de nier son identité juive et de changer de nom, qui était celui de son père : ainsi, Rahel Israilowna devient Ada Igorowna : «Igor. Non pas Israil » ${ }^{45}$. Ces paroles scandent la chronique de la "tête fendue », elles sont de nombreuses fois répétées et variées, exprimant ainsi la dimension traumatisante à la fois de l'événement vécu mais aussi du reniement de l'identité. Énigmatiques au début, le lecteur comprend peu à peu leur sens, de même qu'il saisit mieux l'attitude détachée de la grand-mère vis-à-vis de la famille de son gendre où on parle ouvertement le yiddish, « une langue qu'aucun être raisonnable, aucun homme de lettres n'utiliserait jamais $»{ }^{46}$. Elle observe avec méfiance les rituels juifs, le « lien de la judaïté » qui lie les autres

\footnotetext{
${ }^{43}$ Silke Schwaiger, «Baba Yaga, Schneewittchen und Spaltkopf: Märchenhafte und fantastische Elemente als literarische Stilmittel in Julya Rabinowichs Roman Spaltkopf », Alman Dili ve Edebiyatı Dergisi - Studien zur deutschen Sprache und Literatur, vol. 2, $\mathrm{n}^{\circ} 30 / 2013$, p. 147-163, ici p. 156 : « privat und maßgeschneidert ». En ligne : http://www.journals. istanbul.edu.tr/iuaded/article/view/5000033774. Dernier accès : 17/02/2018.

${ }^{44}$ Yanetta Rybalskaya, «Die matrilineare Familienstruktur in Julya Rabinowichs Roman Spaltkopf oder: Wer ist eigentlich Baba Yaga Girl?», Zeitschrift für interkulturelle Germanistik, vol. 7/1, p. 97-114, ici p. 108.

${ }_{45}$ «Igor. Nicht Israil. » $(S K, 23)$. Cette phrase apparaît dès le premier passage écrit en italiques. Une autre répétition et variation se trouve par exemple p. 47 : «Israil. Nein, Igor ». «Israil. Igor ». « Igor. Nicht Israil »...

${ }^{46}$ «Die neue Verwandtschaft [...] schämt sich nicht, offen Jiddisch, eine Sprache, die kein vernünftiger Mensch, kein Literat je in den Mund nehmen würde, zu sprechen ». $(S K, 97)$.
} 
membres de la famille, alors que le sien est «coupé depuis longtemps $»^{47}$. Cette attitude méprisante ne s'explique donc pas seulement par une différence culturelle et de rang social ouvertement réclamée, mais également par son propre vécu traumatique.

La narratrice, elle-même tardivement mise au courant de son origine juive, ne découvre la véritable identité de sa grand-mère que quand celle-ci se trouve mourante à l'hôpital, alors qu'elle tombe par hasard sur son passeport et voit son vrai nom. Elle n'aura pas d'autres explications. Ce qui reste, c'est le silence en héritage et la nécessité de se confronter à ce refoulé familial. D'une certaine manière, le deuxième récit fortement énigmatique, celui du Spaltkopf, peut très bien être celui que la narratrice invente pour combler les lacunes de l'histoire familiale, récit fait à partir de bribes de vécu, de récits de famille et d'imagination ce qui est le propre de ce que Marianne Hirsch appelle la « postmémoire » des deuxième et troisième générations ${ }^{48}$.

Les trois auteurs dont il était question ici avaient a priori peu de liens avec l'histoire, la religion et la culture juives. Leur exil et leur émigration sont certes dus à l'exclusion et à l'antisémitisme ambiant dans l'Union soviétique des années 1970 ou 1990, mais en général ils ignoraient les racines juives que les parents leur avaient cachées par prudence et qu'ils leur révélaient le plus souvent au moment du départ. Malgré les réticences envers l'Allemagne et l'Autriche, et envers la langue allemande, considérée comme celle des bourreaux, les familles s'y sont installées, dans des conditions souvent difficiles. Les auteurs et leurs protagonistes y sont restés, choisissant l'allemand comme langue d'écriture qui devient, paradoxalement, la langue de transmission de leur vécu et du passé familial. C'est la situation de l'exil qui les fait prendre conscience de leurs racines juives, et la langue allemande véhicule à la fois la quête d'identité conduite dans les récits et la quête de vérité sur leur histoire familiale, pouvant remonter jusqu'à la vie dans le shtetl. On est ici, avec cette génération, à l'opposé de ce dont nous parle Aharon Appelfeld dans le très beau film de Nurith Aviv, D'une langue à l'autre (2004), où l'écrivain dit avoir appris le yiddish "pour chasser l'allemand». Ici, c'est le mouvement inverse : l'allemand sert à retrouver

\footnotetext{
47 « Sie prüft kritisch das jüdische Band, das zwischen den anderen gespannt ist. Das ihre ist lange schon durchgetrennt ». $(S K, 96)$.

${ }^{48}$ Pour une définition concise de ce concept, voir Marianne Hirsch, "Postmémoire », Encyclopédie critique du témoignage et de la mémoire, http://memoriestestimony.com/notice-courte/postmemoire/. Dernier accès : 17/02/2018.
} 
sinon le yiddish, comme dans le cas de Vertlib, du moins l'histoire des grands- et arrière-grands-parents.

Dans leur pratique d'écriture, les auteurs en question trouvent des formes hybrides, c'est notamment le cas de Rabinowich et de Grjasnowa : l'une expérimente avec la forme littéraire, l'autre joue plus sur la langue. En cela, les auteurs enrichissent également la langue et la culture allemandes, ce qui est le propre de toute littérature dite inter- ou transculturelle. Dans Il vortice dei linguaggi, Luca Salza montre comment l'exil, la migration, les vies nomades sont des expériences capables de mobiliser de nouvelles structures narratives, d'autres formes de récits, de nouvelles langues qui se refusent à l'ancrage dans une seule terre, dans une seule langue, une tradition ou un peuple ${ }^{49}$. Selon lui, cette nouvelle langue de l'émigrant ne correspond pas seulement à la langue mal apprise du pays d'accueil, mais à une langue qui surgit grâce aux interférences de l'expérience passée et de la nouvelle réalité dans laquelle il vit. Pour ce rapport à une autre langue, Salza nous rappelle une vieille tradition européenne : «Comme les vieux marins de la Méditerranée, nous pouvons naviguer d'un rivage à l'autre, d'une langue à l'autre ${ }^{50}$. Cela permet, à l'instar des marins, de rencontrer de nouveaux visages et de s'ouvrir à l'altérité. Mais, et c'est le cas des écrivains dont il a été question ici, la découverte de l'altérité peut également déclencher une réflexion sur sa propre identité dont l'autre langue, la nouvelle langue, peut devenir le vecteur.

\footnotetext{
${ }^{49}$ Luca Salza, Il vortice dei linguaggi. Letteratura et migrazione infinita, Messina, Mesogea, 2015, p. 9.

50 Ibid., p. 14 : «Come vecchi marinai del Mediterraneo, possiamo navigare da una costa all'altra, da une lingua all'altra ».
} 